Check for updates

Cite this: RSC Adv., 2017, 7, 51888

Received 6th October 2017

Accepted 23rd October 2017

DOI: 10.1039/c7ra11020k

rsc.li/rsc-advances

\section{Nanostructured spinel manganese cobalt ferrite for high-performance supercapacitors}

\begin{abstract}
Ayman E. Elkholy, (D) ${ }^{\text {ac }}$ F. El-Taib Heakal (D) ${ }^{\mathrm{b}}$ and Nageh K. Allam (D) *c
We report on the synthesis of manganese cobalt ferrite $\left(\mathrm{MnCoFeO}_{4}\right)$ nanoparticles via a simple one-pot coprecipitation method and their characterization through energy-dispersive spectroscopy (EDS), X-ray diffraction (XRD), high-resolution transmission electron microscopy (HR-TEM), Fourier transform infrared (FT-IR) spectroscopy and $\mathrm{N}_{2}$ adsorption/desorption techniques. The $\mathrm{MnCoFeO}_{4}$ supercapacitor showed the maximum specific capacitance of $675 \mathrm{~F} \mathrm{~g}^{-1}$ at a scan rate of $1 \mathrm{mV} \mathrm{s}^{-1}$. Its energy and power densities were $18.85 \mathrm{~W} \mathrm{~h} \mathrm{~kg}^{-1}$ and $337.50 \mathrm{~W} \mathrm{~kg}^{-1}$, respectively, at a current density of $1.5 \mathrm{~A} \mathrm{~g}^{-1}$. The cyclic stability was scrutinized via galvanostatic charging/discharging (GCD) and electrochemical impedance spectroscopy (EIS). The degradation of the supercapacitive performance was only $7.14 \%$ after 1000 GCD cycles, indicating an excellent long-term stability. The equivalent series resistance (ESR) remained nearly constant even after 1000 GCD cycles.
\end{abstract}

\section{Introduction}

Nowadays, the worldwide community suffers from serious environmental problems arising from an excessive combustion of fossil fuels. ${ }^{1}$ Consequently, many researchers have paid great attention to manipulate the clean and sustainable energy sources as well as efficient energy conversion and storage technologies. ${ }^{2}$ Batteries and supercapacitors are the typical devices used for the storage of electrical energy. Both of these store electricity through electrochemical processes. ${ }^{3}$ Compared to the batteries, the supercapacitors are characterized by higher power density, rapid charge/discharge, and long-life service. Despite the advantages of high power density and high cycle life of supercapacitors, their intrinsically low energy density (i.e. the amount of energy stored per unit weight) has limited them from widespread commercial applications in comparison to batteries. To this end, increasing their energy density requires an extensive research and development, ${ }^{4,5}$ including the merge of batteries and supercapacitors. ${ }^{6}$

According to their active materials, supercapacitors (SCs) are classified into two main types: electrochemical double layer capacitors (DCs) and electrochemical pseudocapacitors (PCs). ${ }^{7,8}$ The active material in the DCs is composed of carbonaceous (carbon-based) materials, such as activated carbon, graphite, and graphene. Energy is stored physically within a DC via charge accumulation across the electrode/electrolyte

\footnotetext{
${ }^{a}$ Department of Analysis and Evaluation, Egyptian Petroleum Research Institute, 11727 Cairo, Egypt

${ }^{b}$ Chemistry Department, Faculty of Science, Cairo University, 12613 Giza, Egypt

${ }^{c}$ Energy Materials Laboratory, School of Sciences and Engineering, The American University in Cairo, 11835 New Cairo, Egypt.E-mail: nageh.allam@aucegypt.edu
}

interface. ${ }^{8,9}$ As for PCs, the active material is primarily composed of a transition metal (TM) oxide, a TM nitride or a conducting polymer. Energy is stored electrochemically within a PC via the reversible interfacial redox reactions in TMs or via ion intercalation throughout the electrode in conducting polymers. ${ }^{5,10,11}$ Due to the intrinsic low specific capacitance $\left(C_{\mathrm{sp}}\right)$ and low energy density stored in the current SCs, it is vital to explore new materials that simultaneously exhibit high $C_{\mathrm{sp}}$ as well as high conductivity. ${ }^{4} \mathrm{TMs}$ are characterized by being cost-effective and displaying multiple oxidation states ${ }^{7}$ in addition to their fast and reversible faradaic redox reactions. ${ }^{12}$ Therefore, TMs are engrossed as the electrode materials for supercapacitor applications because of their outstanding electrochemical performance. ${ }^{13}$ In addition, TM oxides can provide a higher theoretical $C_{\mathrm{sp}}$ than that of the conventional carbon-based materials and a better electrochemical stability than that of the polymeric materials. ${ }^{7}$ Among them, $\mathrm{RuO}_{2}$ has been widely investigated as a promising candidate because of its better conductivity and high $C_{\mathrm{sp}}$; however, it is limited by its high cost, rarity, ${ }^{13,14}$ and toxicity. ${ }^{15}$ Alternative inorganic electrode materials such as $\mathrm{MnO}_{2},{ }^{16} \mathrm{Co}_{3} \mathrm{O}_{4},{ }^{17} \mathrm{NiO},{ }^{18} \mathrm{~V}_{2} \mathrm{O}_{5} / \mathrm{VO}_{4},{ }^{19} \mathrm{WO}_{3},{ }^{20}$ and $\mathrm{Fe}_{2} \mathrm{O}_{3}{ }^{21}$ have been intensively investigated in SC applications owing to their wide availability, chemical stability, mechanical strength, safety, and eco-friendliness..$^{15}$ Recently, ferrites as SC materials have been explored by researchers due to their various redox states, electrochemical stability as well as remarkable magnetic, catalytic, optical, and electrical properties. ${ }^{22}$ Ferrite-based materials have been synthesized in diverse nanostructured forms, including nanoparticles, ${ }^{23}$ nanotubes,${ }^{24}$ nanofibers, ${ }^{20}$ nanowires, ${ }^{25,26}$ nanorods,${ }^{10}$ nanoflakes, ${ }^{17,27}$ nanomesh arrays, ${ }^{14}$ nanosheets, ${ }^{17}$ and hollow structures. ${ }^{28}$ Ferrites can be synthesized via various synthesis techniques 
including sol-gel method, ${ }^{12}$ co-precipitation method, ${ }^{29}$ template method, ${ }^{24}$ solvothermal method, ${ }^{30}$ microwave-assisted method, ${ }^{10,13}$ electrodeposition method, ${ }^{27}$ chemical spray method, ${ }^{15}$ spray-pyrolysis method $^{15}$ and hydrothermal method. ${ }^{14}$ Among these techniques, the co-precipitation method provides a simple route for the one-pot synthesis and the reaction conditions are mild and simple..$^{13}$

Usually, the general formula for a spinel is $\mathrm{AB}_{2} \mathrm{O}_{4}$, where $\mathrm{A}$ refers to a divalent metal ion $\left(\mathrm{M}^{2+}\right)$ and $\mathrm{B}$ refers to a trivalent metal ion $\left(\mathrm{M}^{3+}\right)$. In a normal spinel structure, the A ions occupy the tetrahedral sites and the $\mathrm{B}$ ions occupy the octahedral sites. ${ }^{31}$ Spinel ferrites, $\mathrm{MFe}_{2} \mathrm{O}_{4}$ or $\mathrm{MM}^{\prime} \mathrm{FeO}_{4}$ (where $\mathrm{M}$ or $\mathbf{M}^{\prime}=$ $\mathrm{Mn}, \mathrm{Co}, \mathrm{Ni}, \mathrm{Zn}, \mathrm{Cu}$, etc.), are fascinating materials owing to their impressive magnetic, electrical, and optical properties in addition to their ability to exhibit different redox states and electrochemical stability. In spinel ferrites, the divalent metal ion $\left(\mathrm{M}^{2+}\right)$ occupies the tetrahedral site and the trivalent metal ion $\left(\mathrm{M}^{\prime 3+}\right.$ or $\left.\mathrm{Fe}^{3+}\right)$ occupies the octahedral position. ${ }^{\mathbf{1 2}}$ According to Bernard et al. ${ }^{32}$ and Kulkarni, ${ }^{33} \mathrm{Mn}$ atoms in $\mathrm{MnCoFeO}_{4}$ prefer to occupy the tetrahedral positions, while Co atoms prefer to occupy the octahedral sites. Besides, the valence states of $\mathrm{Mn}$, Co, and Fe are majorly (+II), (+III), and (+III), respectively. In contrast, Martens ${ }^{34}$ has studied the magneto-optical properties of $\mathrm{Mn}_{x} \mathrm{CoFe}_{2-x} \mathrm{O}_{4}$ (where $x=0,0.5$, and 1.0) prepared using a conventional ceramic technology with a final sinter treatment in oxygen for $24 \mathrm{~h}$ at $1200-1300{ }^{\circ} \mathrm{C}$ and found that $\mathrm{Mn}$ has the oxidation state $(+\mathrm{III})$ and occupied the octahedral sites, while $\mathrm{Co}$, in the form of $\mathrm{Co}^{2+}$, could occupy both octahedral and tetrahedral positions with the latter being more favorable. These observations were confirmed by two later studies based on submicron $\mathrm{Mn}_{x} \mathrm{CoFe}_{2-x} \mathrm{O}_{4}$ spinel ferrites by Chassaing et $a .^{35}$ and Laarj and Kacim. ${ }^{36}$ In both studies, $\mathrm{Mn}_{x} \mathrm{CoFe}_{2-x} \mathrm{O}_{4}$ spinels were prepared from the oxalic precursors and subjected to annealing treatment between $600{ }^{\circ} \mathrm{C}$ and $700{ }^{\circ} \mathrm{C}$.

Numerous binary TM ferrites have been investigated for supercapacitor applications such as $\mathrm{ZnFe}_{2} \mathrm{O}_{4},{ }^{17,30} \mathrm{CoFe}_{2} \mathrm{O}_{4},{ }^{14}$ $\mathrm{MnFe}_{2} \mathrm{O}_{4},{ }^{37} \mathrm{CuFe}_{2} \mathrm{O}_{4},{ }^{26}$ and $\mathrm{SnFe}_{2} \mathrm{O}_{4} \cdot{ }^{10}$ However, few research studies were carried out on ternary TM ferrites as the electrodes for supercapacitor applications as listed in Table 1.

Herein, $\mathrm{MnCoFeO}_{4}$ was synthesized in the form of nanoparticles via the co-precipitation method and characterized by EDS, XRD, TEM, FT-IR analyses, and $\mathrm{N}_{2}$ adsorption/desorption. Its supercapacitive performance in $6 \mathrm{M} \mathrm{KOH}$ was investigated via cyclic voltammetry (CV) and galvanostatic charging/ discharging (GCD). The cyclic stability was studied via GCD and electrochemical impedance spectroscopy (EIS). The materials showed an exceptional $C_{\mathrm{sp}}$ as compared to the $C_{\mathrm{sp}}$ of those reported in the literature.

\section{Experimental methods}

\subsection{Preparation of $\mathrm{MnCoFeO}_{4}$}

To obtain $\mathrm{MnCoFeO}_{4}, 0.005$ mole of $\mathrm{MnCl}_{2} \cdot 4 \mathrm{H}_{2} \mathrm{O}$ was dissolved in $50 \mathrm{ml}$ deionized water, 0.005 mole of $\mathrm{CoCl}_{2} \cdot 6 \mathrm{H}_{2} \mathrm{O}$ was dissolved in $50 \mathrm{ml}$ deionized water, and 0.01 mole of anhydrous $\mathrm{FeCl}_{3}$ was dissolved in $250 \mathrm{ml}$ deionized water. The three solutions were mixed together with continuous stirring and kept at $65{ }^{\circ} \mathrm{C}$ until thermal equilibrium was achieved. Then, $100 \mathrm{ml}$ of $0.8 \mathrm{M} \mathrm{NaOH}$ solution was added dropwise to the mixed solution with continuous stirring and a black precipitate was formed. The formed precipitate was separated from the liquid phase and washed several times with deionized water via centrifugation until neutrality. The neutrality of the decanted aliquot was checked using phenolphthalein indicator. Finally, the washed precipitate was dried at $100{ }^{\circ} \mathrm{C}$.

\subsection{Characterization of $\mathrm{MnCoFeO}_{4}$}

Elemental composition was identified by means of energydispersive spectroscopy (EDS) using an Oxford EDS detector. The structural characteristics of the prepared material were investigated via X-ray diffraction (XRD) using a PANalytical $\mathrm{X}$ 'pert PRO diffractometer with a $\mathrm{Cu} \mathrm{K} \alpha$ radiation. The particle morphology of the prepared material was studied via highresolution transmission electron microscopy (HR-TEM) using a JEOL JEM-2100 (Japan) electron microscope, operating at an accelerated voltage of $200 \mathrm{keV}$. Prior to the analysis, the sample was dispersed in ethanol using a probe sonicator and then a drop of the much-diluted sample solution was deposited on a carbon-coated copper grid and allowed to be evaporated at room temperature. Fourier transform infrared (FT-IR) spectroscopy was performed in the range of $400-4000 \mathrm{~cm}^{-1}$ to determine the metal oxide peaks using a PerkinElmer Spectrum One spectrophotometer. The adsorption capability of the prepared material and its specific surface area were investigated by $\mathrm{N}_{2}$ gas adsorption/desorption (at $77 \mathrm{~K}$ ) and BrunauerEmmett-Teller (BET) analysis, respectively, using a Quantachrome NOVA Station A (version 11.03).

\subsection{Electrochemical measurements}

A working electrode was prepared using a homogenous slurry of $\mathrm{MnCoFeO}_{4}$ with carbon black acting as a conductive additive

Table 1 Supercapacitive performance of $\mathrm{MnCoFeO}_{4}$ compared with some other ferrite-based materials

\begin{tabular}{|c|c|c|c|c|}
\hline Material & $C_{\mathrm{sp}}\left(\mathrm{F} \mathrm{g}^{-1}\right)$ & $\mathrm{ED}\left(\mathrm{W} \mathrm{h} \mathrm{kg}^{-1}\right)$ & $\mathrm{PD}\left(\mathrm{W} \mathrm{kg}^{-1}\right)$ & Ref. \\
\hline $\mathrm{CuCoFe}_{2} \mathrm{O}_{4}$ & 76.9 & 7.90 & 1711.95 & 12 \\
\hline $\mathrm{NiCoFe}_{2} \mathrm{O}_{4}$ & 50.0 & 4.79 & 1426.23 & 12 \\
\hline $\mathrm{CuCoFe}_{2} \mathrm{O}_{4}$ & 397.0 & 3.53 & 198.50 & 49 \\
\hline $\mathrm{Al}_{x} \mathrm{Cu}_{y} \mathrm{Co}_{z} \mathrm{Fe}_{2} \mathrm{O}_{4}(x+y+z=1)$ & $256.0-540.0$ & $0.58-8.42$ & $128.00-270.00$ & 49 \\
\hline $\mathrm{MnCoFeO}_{4}$ & 670.0 & 3.15 & 2250.00 & This work \\
\hline
\end{tabular}


and poly(vinylidene difluoride) acting as a binder in a DMF solvent with a weight ratio of $70: 20: 10$. The prepared slurry was stirred for about $48 \mathrm{~h}$ at room temperature, then coated onto a part of a $1 \mathrm{~cm} \times 2 \mathrm{~cm}$ chip of nickel foam (as a supporter and current collector) and dried at $60{ }^{\circ} \mathrm{C}$. The supercapacitive performance of the prepared $\mathrm{MnCoFeO}_{4}$ electrode was investigated using a three-electrode cell containing the working electrode (the prepared electrode under study), a counter electrode (a Pt coil) and a reference electrode (a saturated calomel electrode, SCE) in a $6 \mathrm{M} \mathrm{KOH}$ solution at room temperature. The electrochemical measurements involved cyclic voltammetry (CV), galvanostatic charging/discharging (GCD), and electrochemical impedance spectroscopy (EIS) using the electrochemical workstation (CHI 760D, CH Instruments, U.S.A.). CV measurements were performed within the potential window of $0-0.45 \mathrm{~V}$ at different scan rates (from 1 to $100 \mathrm{mV} \mathrm{s}^{-1}$ ). GCD measurements were carried out at different current densities (1.5-10 $\left.\mathrm{A} \mathrm{g}^{-1}\right)$ within the same potential window. The EIS measurements were performed in the frequency range of $100 \mathrm{kHz}$ to $0.1 \mathrm{~Hz}$ at the steady-state open circuit potential ( $0.261 \mathrm{~V}$ vs. SCE) with a sinusoidal perturbation amplitude of $10 \mathrm{mV}$. EIS parameters were derived using EC-Lab V10.40 software.

The specific capacitance $\left(C_{\mathrm{sp}}, \mathrm{F} \mathrm{g}^{-1}\right)$ was then calculated from data obtained from CV and GCD measurements according to eqn (1) ${ }^{10,22}$ and (2) ${ }^{14,17,38}$ respectively.

$$
\begin{gathered}
C_{\mathrm{sp}}=\frac{\int I(\mathrm{~d} V)}{m v \Delta V} \\
C_{\mathrm{sp}}=\frac{I \Delta t}{m \Delta V}
\end{gathered}
$$

where $I$ is the response current (A), $m$ is the mass of active material $(\mathrm{g}), v$ is the scan rate $\left(\mathrm{mV} \mathrm{s}^{-1}\right), \Delta V$ is the potential window $(\mathrm{V})$, and $\Delta t$ is the discharge time (s). The coulombic efficiency $(\eta)$ was calculated from GCD measurements as follows: $:^{39,40}$

$$
\eta=\frac{t_{\mathrm{d}}}{t_{\mathrm{c}}} \times 100
$$

where $t_{\mathrm{c}}$ and $t_{\mathrm{d}}$ are the times of charging and discharging with the same current density, respectively. The energy density and the power density of the $\mathrm{MnCoFeO}_{4}$ supercapacitor can be calculated from eqn (5) and (6) $\mathbf{1 4 , 4 0 - 4 2}^{\mathbf{1}}$

$$
E=\frac{1}{2} C_{\mathrm{sp}} \Delta V^{2}
$$

eqn (4) can be reformed as follows:

$$
\begin{gathered}
E=\frac{1}{2} \frac{I \Delta V t}{m} \\
P=\frac{E}{t}
\end{gathered}
$$

where $I$ is the response current (A), $m$ is the mass of active material $(\mathrm{kg}), \Delta V$ is the potential window $(\mathrm{V})$, and $t$ is the discharge time $(\mathrm{h})$.

\section{Results and discussion}

\subsection{Material characterization}

Fig. 1a demonstrates the EDS spectrum of the prepared $\mathrm{MnCoFeO}_{4}$, showing the peaks of $\mathrm{Mn}, \mathrm{Co}, \mathrm{Fe}$, and O elements. The presence of these elements in the formed precipitate confirmed that $\mathrm{Mn}$, Co, and Fe have co-precipitated simultaneously in the oxide form. The elemental composition obtained from EDS analysis reveals that the atomic percentages of $\mathrm{Mn}$, Co, Fe, and $\mathrm{O}$ are $10.14 \%, 9.81 \%, 18.75 \%$, and $61.30 \%$, respectively. As a result, their atomic ratio is approximately $1: 1: 2: 6$. Fig. 1b shows the XRD pattern of the prepared material. All diffraction peaks are coincident to the Miller
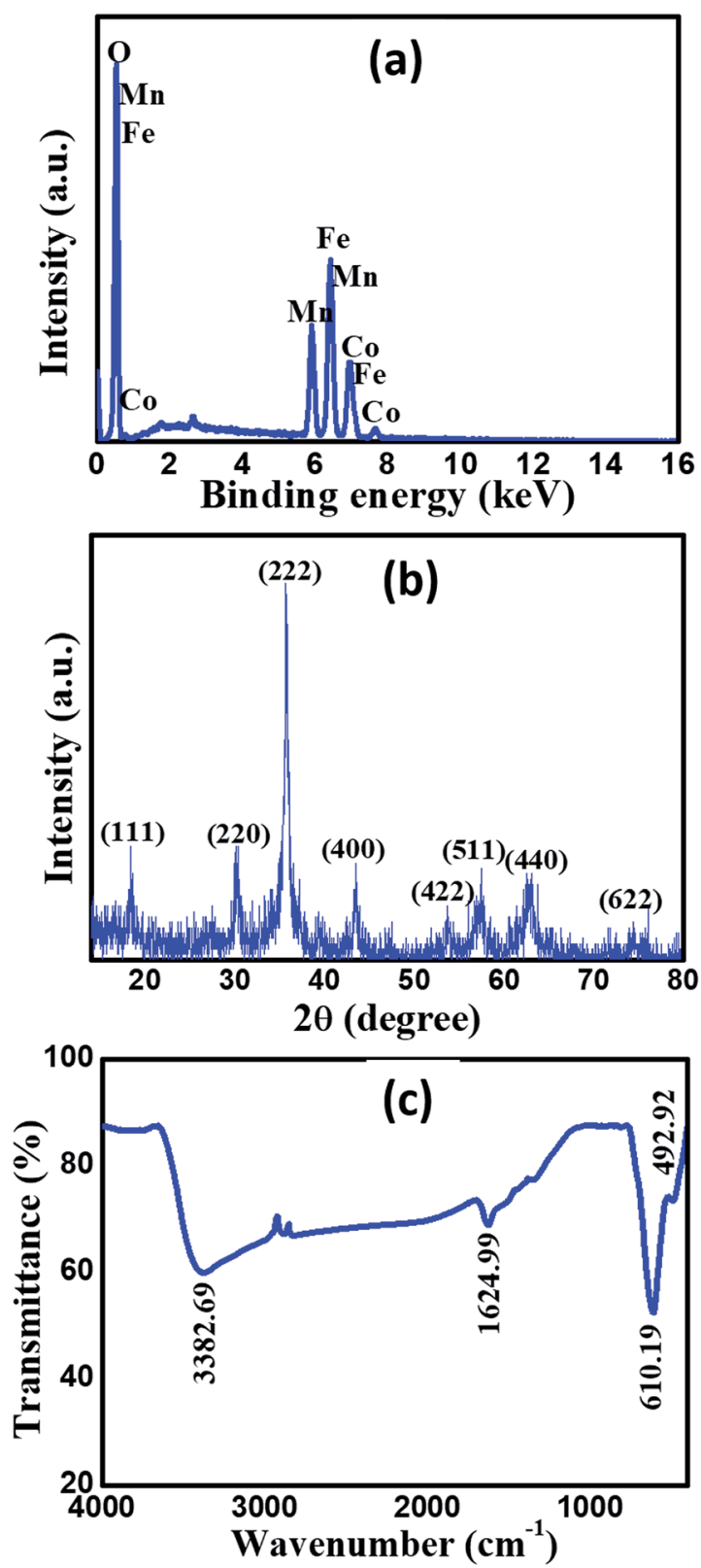

Fig. 1 (a) EDS spectrum, (b) XRD pattern, and (c) FT-IR spectrum for the as-prepared $\mathrm{MnCoFeO}_{4}$. 
indices (111), (220), (222), (400), (422), (511), (440) and (622) of the standard data for $\mathrm{MnCoFeO}_{4}$ (cubic, space group: $F d \overline{3} m$, ICDD card number: 04-010-1895), which confirms the formation of $\mathrm{MnCoFeO}_{4}$ with the spinel structure. Fig. 1c displays the FT-IR spectrum of $\mathrm{MnCoFeO}_{4}$ recorded in the frequency range 4000-400 $\mathrm{cm}^{-1}$. The lower-frequency band $\left(493 \mathrm{~cm}^{-1}\right)$ is assigned to the octahedral groups $\left(\left[\mathrm{Fe}^{3+}-\mathrm{O}^{2-}\right]\right.$ and $\left.\left[\mathrm{Mn}^{3+}-\mathrm{O}^{2-}\right]\right)$, while the higher-frequency band $\left(610 \mathrm{~cm}^{-1}\right)$ is assigned to the stretching of the tetrahedral groups $\left(\left[\mathrm{Co}^{2+}-\mathrm{O}^{2-}\right]\right)$ present in the spinel ferrite. ${ }^{\mathbf{1 2}}$ The FT-IR absorption bands appearing at $3383 \mathrm{~cm}^{-1}$ and $1625 \mathrm{~cm}^{-1}$ are referred to adsorbed water molecules. ${ }^{41}$

Fig. 2 shows the HR-TEM images of the as-fabricated $\mathrm{MnCoFeO}_{4}$, indicating the formation of nanoparticles with sizes ranging $c a$. 30-40 nm. Fig. 3a shows the $\mathrm{N}_{2}$ adsorption/ desorption isotherm for the as-synthesized $\mathrm{MnCoFeO}_{4}$. According to the IUPAC classification of gas adsorption/ desorption isotherms, ${ }^{43}$ the obtained $\mathrm{N}_{2}$ adsorption/ desorption isotherm is of type IV, which is characteristic to the mesoporous materials. The characteristic feature of this isotherm is its hysteresis loop, which arises when the adsorption and desorption curves do not coincide and is associated with the capillary condensation taking place in the mesopores. Moreover, the initial part, where adsorption/desorption curves are coincident, is attributed to the monolayer-multilayer adsorption. These features confirm the mesoporous structure of the prepared $\mathrm{MnCoFeO}_{4}$. It is worth mentioning that porous materials are generally classified according to their pore diameter $(d)$ into three categories: macroporous $(d>50 \mathrm{~nm})$, mesoporous $(d=2-50 \mathrm{~nm})$, and microporous materials $(d<2 \mathrm{~nm}){ }^{5}$ Fig. $3 \mathrm{~b}$ shows the pore diameter distribution curve obtained by the Barrett-Joyner-Halenda (BJH) method using the desorption branch of the nitrogen isotherm. ${ }^{17}$ It reveals an average pore diameter of $10.036 \mathrm{~nm}$. The BET method likely yields a value of actual surface area if the isotherm is either of type II or type IV. ${ }^{43}$ Hence, the surface area obtained from multipoint BET is noted to be $104.963 \mathrm{~m}^{2} \mathrm{~g}^{-1}$.

\subsection{Electrochemical characterization}

3.2.1. Specific capacitance. The electrochemical capacitive performance of the as-fabricated $\mathrm{MnCoFeO}_{4}$ was examined via $\mathrm{CV}$ and GCD measurements. The CV curves of $\mathrm{MnCoFeO}_{4}$ were recorded at different scan rates in the potential range of $0-0.45$ $\mathrm{V}_{\mathrm{SCE}}$ in $6 \mathrm{M} \mathrm{KOH}$ (Fig. 4a). The CV curves show faradic-type capacitive features, where two peaks appear at $\sim 0.38 \mathrm{~V}$ and $\sim 0.19 \mathrm{~V}$ for the oxidation and reduction processes, respectively. The specific capacitance $\left(C_{\mathrm{sp}}\right)$ obtained from $\mathrm{CV}$ measurements is dependent on the scan rate, where the peak current density $\left(I_{\mathrm{p}}, \mathrm{A} \mathrm{g}^{-1}\right)$ of $\mathrm{MnCoFeO}_{4}$ clearly increases as a function of scan rate. The maximum $C_{\mathrm{sp}}\left(675 \mathrm{~F} \mathrm{~g}^{-1}\right)$ is obtained at the slowest scan rate $\left(1 \mathrm{mV} \mathrm{s}^{-1}\right)$, while $C_{\mathrm{sp}}$ calculated at $100 \mathrm{mV} \mathrm{s}^{-1}$ is $238.81 \mathrm{~F} \mathrm{~g}^{-1}$ (the inset of Fig. 4a). This could be attributed to the existence of a large Ohmic resistance at large scan rates. ${ }^{37}$ In addition, the electrolyte ions do not have a sufficient time, at high rates of potential scanning, to entirely diffuse through the electrode nanopores wherever the faradaic reactions occur. ${ }^{8}$ Also, this behavior suggests that there are parts of the electrode surface inaccessible at high rates of charging/discharging. ${ }^{44}$ Therefore, electrical energy is electrochemically stored in an efficient manner in the $\mathrm{MnCoFeO}_{4}$ supercapacitor at a slower scan rate.

Moreover, Fig. 4a demonstrates the pairs of distinct and broad redox peaks corresponding to the redox transitions of $\mathrm{Co}^{3+} / \mathrm{Co}^{2+}$ and $\mathrm{Mn}^{3+} / \mathrm{Mn}^{2+}$. The redox peaks of $\mathrm{Co}^{3+} / \mathrm{Co}^{2+}$ and $\mathrm{Mn}^{3+} / \mathrm{Mn}^{2+}$ seem to be merging together because their standard electrode potentials are comparable (1.92 and $1.50 \mathrm{~V}_{\mathrm{NHE}}$, respectively ${ }^{45}$ ). The probable processes associated with the capacitive behavior of $\mathrm{MnCoFeO}_{4}$ can be related to the presence of two redox systems. The redox reactions related to both systems could proceed according to eqn $(7)^{\mathbf{4 6}}$ and $(8)^{\mathbf{4 2}}$, respectively.

$$
\begin{array}{r}
\mathrm{Co}^{2+} / \mathrm{Co}^{3+} \text { system: } \mathrm{CoFe}_{2} \mathrm{O}_{4}+\mathrm{OH}^{-}+\mathrm{H}_{2} \mathrm{O} \leftrightarrow \\
2 \mathrm{FeOOH}+\mathrm{CoOOH}+\mathrm{e}
\end{array}
$$
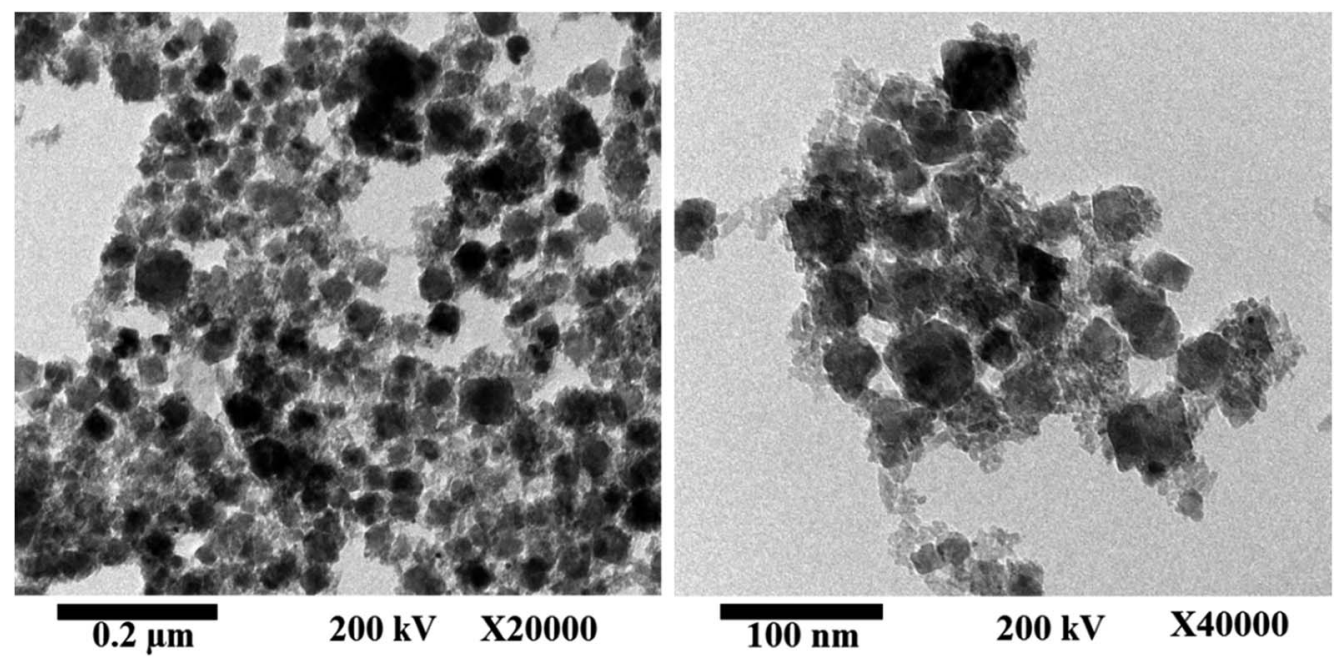

Fig. $2 \mathrm{HR}$-TEM images of the as-prepared $\mathrm{MnCoFeO}_{4}$. 

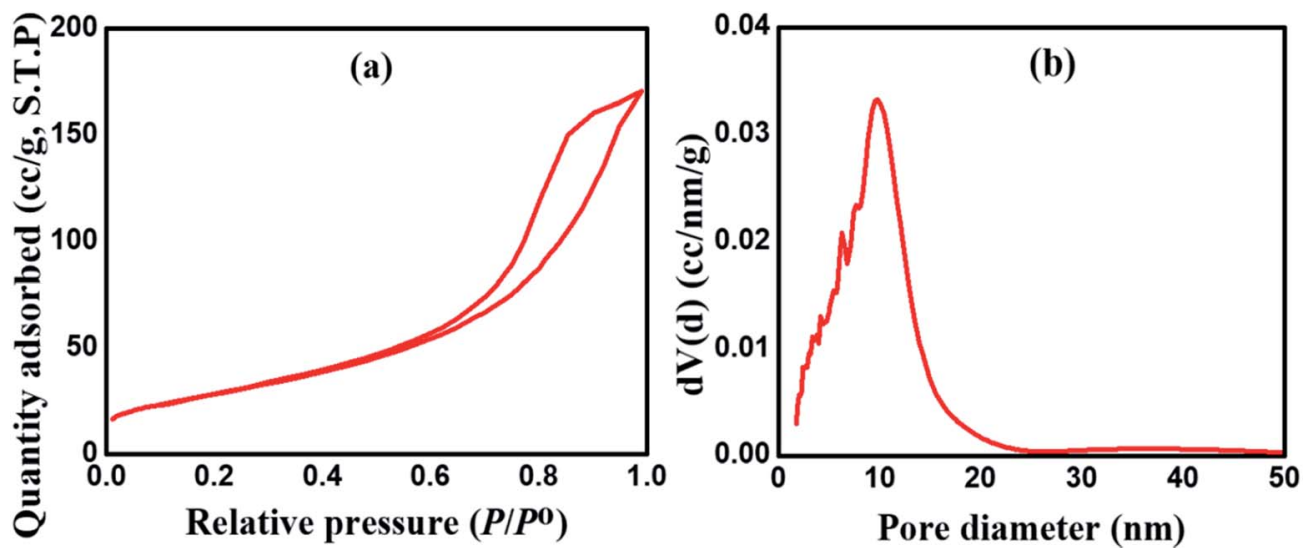

Fig. 3 (a) $\mathrm{N}_{2}$ adsorption/desorption isotherm and (b) $\mathrm{BJH}$ pore size distribution for the as-prepared $\mathrm{MnCoFeO}_{4}$.
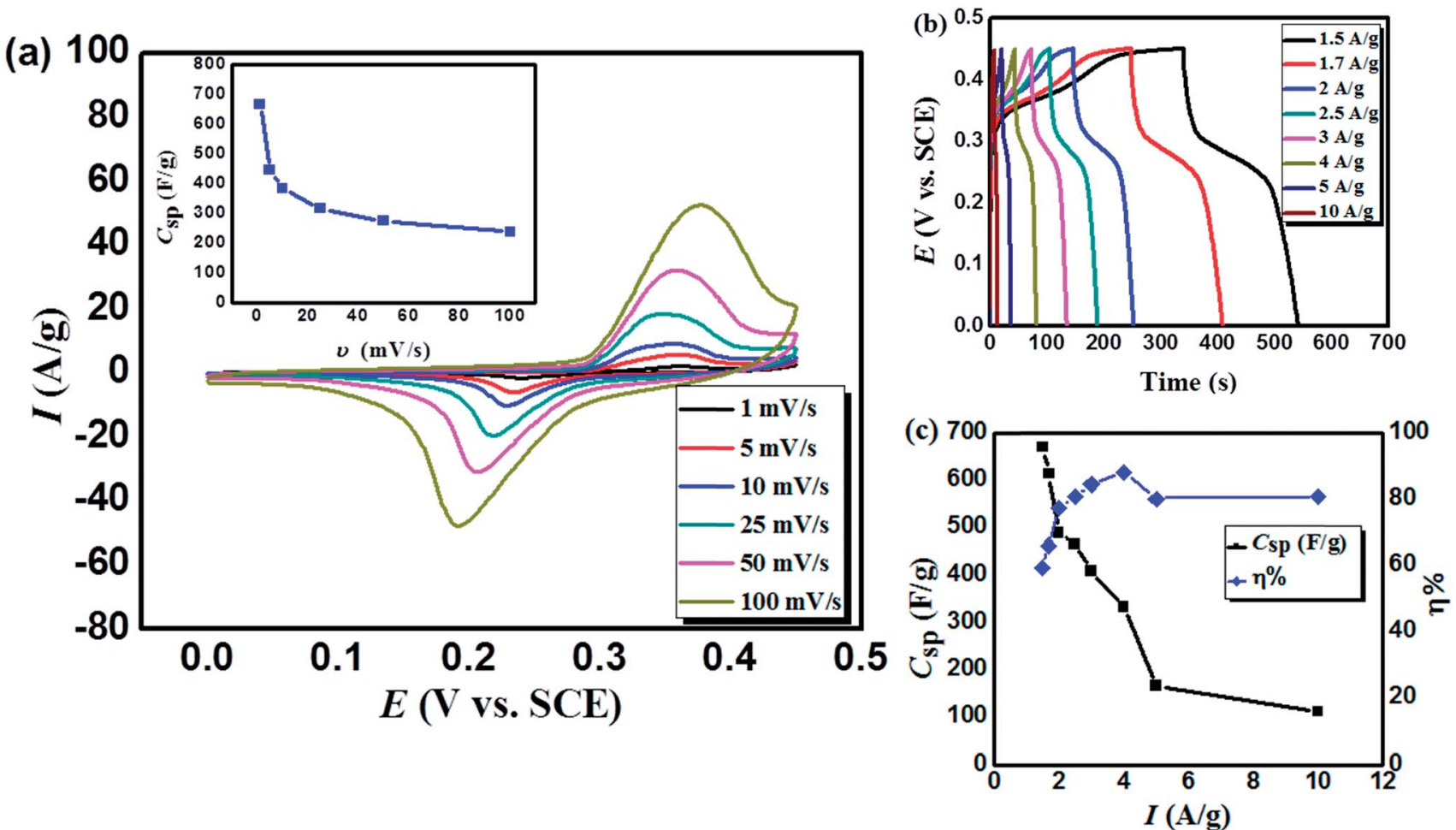

Fig. 4 (a) $\mathrm{CV}$ curves recorded for as-prepared $\mathrm{MnCoFeO}_{4}$ at different scan rates in the potential range of $0-0.45 \mathrm{~V}_{\mathrm{SCE}}$ in the $6 \mathrm{M} \mathrm{KOH}$ electrolyte and the inset shows the dependence of the specific capacitance on the scan rate, (b) Galvanostatic charge/discharge curves recorded for $\mathrm{MnCOFeO}_{4}$, and (c) the calculated $C_{\text {sp }}$ and $\eta \%$ at different current densities in the potential range of $0-0.45 \mathrm{~V}$ SCE in the $6 \mathrm{M} \mathrm{KOH}$ electrolyte.

$\mathrm{Mn}^{2+} / \mathrm{Mn}^{3+}$ system: $\mathrm{MnFe}_{2} \mathrm{O}_{4}+\mathrm{OH}^{-}+\mathrm{H}_{2} \mathrm{O} \leftrightarrow$

$$
2 \mathrm{FeOOH}+\mathrm{MnOOH}+\mathrm{e}
$$

The CV profiles (at different scan rates) demonstrate the same behavior, revealing the reversibility of the redox reactions. In addition, with the increase in the scan rate, both oxidation and reduction peaks shift towards more anodic and cathodic directions, respectively. For example, upon increasing the scan rate from 1 to $100 \mathrm{mV} \mathrm{s}^{-1}$, the anodic peak potential $\left(E_{\mathrm{p}, \mathrm{a}}\right)$ increased from 0.358 to $0.378 \mathrm{~V}_{\mathrm{SCE}}$, while the anodic peak current $\left(I_{\mathrm{p}, \mathrm{a}}\right)$ increased from 2.063 to $52.494\left(\mathrm{~A} \mathrm{~g}^{-1}\right)$. This effect results from the unavoidable overpotential due to the increase in the internal diffusion resistance and $I_{\mathrm{p}}$ increases with $v$ due to the fast interfacial kinetics. ${ }^{\mathbf{1 4}}$

Galvanostatic charge/discharge (GCD) measurement is an accurate technique for determining the electrochemical performance of supercapacitors particularly for those based on pseudo-capacitance. ${ }^{40}$ Fig. $4 \mathrm{~b}$ displays the GCD curves recorded for $\mathrm{MnCoFeO}_{4}$ in $6 \mathrm{M} \mathrm{KOH}$. Unlike the GCD curves of carbonbased materials exhibiting a semi-triangular shape, where their capacitances are mainly attributed to a pure electric double layer capacitance, the GCD curves of $\mathrm{MnCoFeO}_{4}$ show 
deviations from linearity due to its pseudocapacitive nature..$^{22,47}$ Clearly, the $\mathrm{MnCoFeO}_{4}$ electrode demonstrates higher charging and discharging times as the current density decreases, resulting in higher values of specific capacitance (Fig. 4c). For example, on decreasing the current density from $10 \mathrm{~A} \mathrm{~g}^{-1}$ to $1.5 \mathrm{~A} \mathrm{~g}^{-1}, C_{\mathrm{sp}}$ increases from 112 to $670 \mathrm{~F} \mathrm{~g}^{-1}$. Furthermore, the columbic efficiency $(\eta \%)$ increases with the current density up to $89 \%$ at $4 \mathrm{~A} \mathrm{~g}^{-1}$, after which it becomes constant $(\sim 81 \%)$.
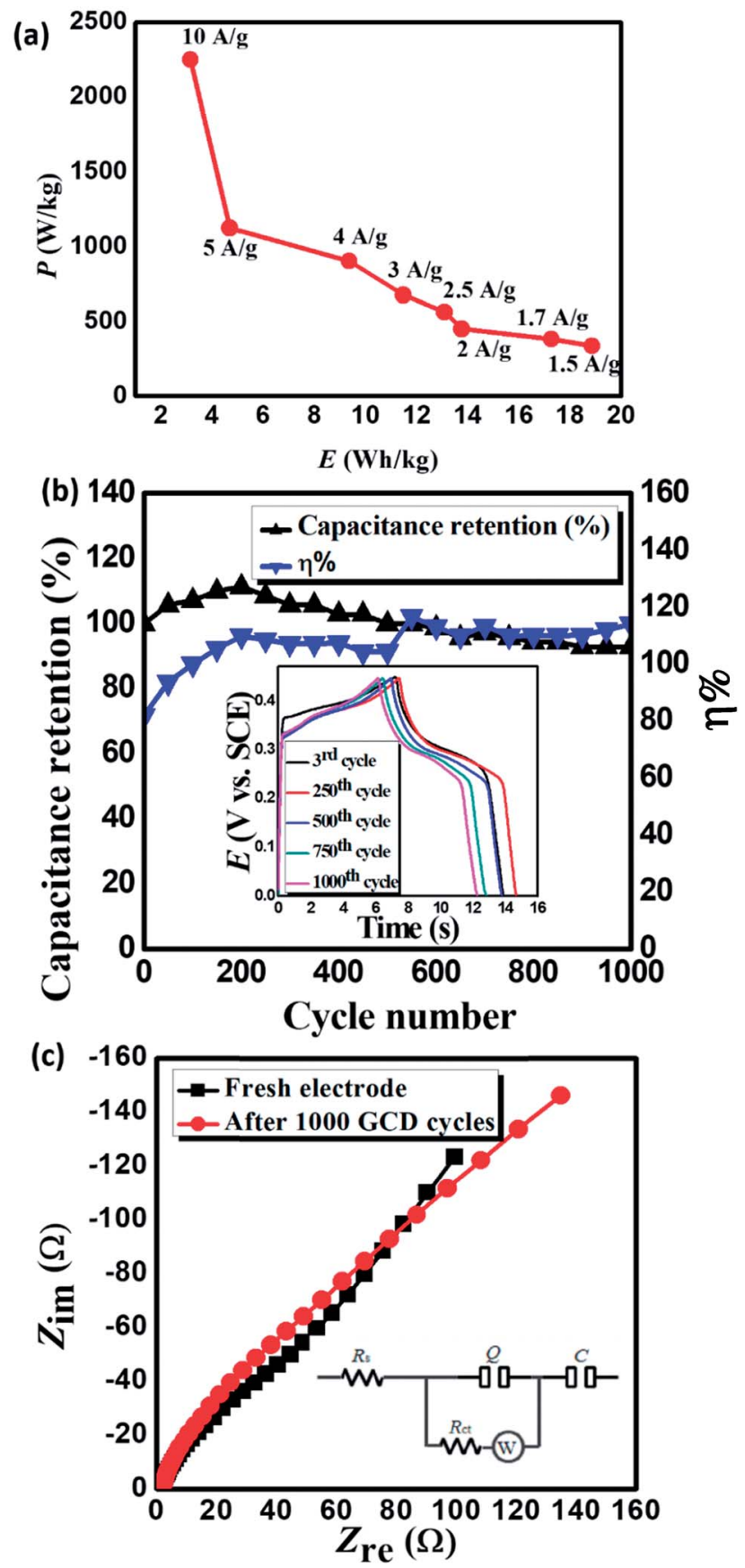

Fig. 5 (a) Ragone plot, (b) cycling stability curves, and (c) Nyquist spectra recorded for $\mathrm{MnCoFeO}_{4}$ before and after 1000 galvanostatic charge/discharge (GCD) cycles.
Power density (PD) and energy density (ED) are two important parameters for the evaluation of the electrochemical performance of supercapacitors and electrode materials. ${ }^{40}$ Fig. 5a displays the Ragone plot of $\mathrm{MnCoFeO}_{4}$ obtained from GCD measurements ${ }^{48}$ with the current densities ranging from 1.5 to $10 \mathrm{~A} \mathrm{~g}^{-1}$. Fig. $5 \mathrm{a}$ demonstrates that the ED stored in $\mathrm{MnCoFeO}_{4}$ and its corresponding PD (at a current density of $1.5 \mathrm{~A} \mathrm{~g}^{-1}$ ) are $18.85 \mathrm{~W} \mathrm{~h} \mathrm{~kg}^{-1}$ and $337.50 \mathrm{~W} \mathrm{~kg}^{-1}$, respectively. On the other hand, at a current density of $10 \mathrm{~A} \mathrm{~g}^{-1}$, ED and PD values are $3.15 \mathrm{~W} \mathrm{~h} \mathrm{~kg}^{-1}$ and $2250.00 \mathrm{~W} \mathrm{~kg}^{-1}$, respectively. Table 1 depicts the supercapacitive performance parameters, including $C_{\mathrm{sp}}$, $\mathrm{PD}$, and $\mathrm{ED}$ for $\mathrm{MnCoFeO}_{4}$ and some ternary and quaternary TM ferrites. It should be noted that $\mathrm{MnCoFeO}_{4}$ has an excellent $C_{\mathrm{sp}}$ and PD in comparison to those of the previously investigated materials.

3.2.2. Cycling stability. Cycling stability is another important factor that determines the efficiency and durability of supercapacitors, ${ }^{37}$ which can be expressed in terms of capacitance retention (\%) and coulombic efficiency $(\eta \%) .{ }^{40}$ Fig. 5b illustrates the capacitance retention (\%) and $\eta \%$ of the prepared supercapacitor recorded during 1000 continuous GCD cycles at a high current density $\left(10 \mathrm{~A} \mathrm{~g}^{-1}\right)$. The capacitance retention (\%) increases up to 200 cycles and reaches $111.43 \%$. Following this, it starts to decrease and reaches $\sim 92.86 \%$ after 1000 GCD cycles, indicating an excellent long-term stability. In addition, the coulombic efficiency increases with time and reaches $114 \%$ after $1000 \mathrm{GCD}$ cycles. The excellent stability of $\mathrm{MnCoFeO}_{4}$ could be attributed to the synergistic effects of the three transition metals.

EIS is a powerful tool used to investigate the features of an electrode/electrolyte interface as supercapacitors by evaluating the frequency behavior and equivalent series resistance (ESR). ${ }^{37}$ EIS offers information about the internal resistance of the electrode material and the resistance between the electrode and the electrolyte. ${ }^{40}$ EIS spectra (Fig. 5c) display a depressed semicircle in the high-frequency region linked to an inclined straight line in the low-frequency region. The slope of the inclined line is decreased after 1000 GCD cycles. The equivalent circuit used to analyze EIS spectra is depicted in the inset of Fig. 5 c. ${ }^{48}$

The time constant $\left(Q R_{\mathrm{ct}} W\right)$ represents the depressed semicircle in the high-frequency region, where $R_{\mathrm{ct}}$ is the chargetransfer resistance caused by the faradaic process and $Q$ is a constant phase element representing the non-ideal double-

Table 2 EIS parameters calculated for the $\mathrm{MnCoFeO}_{4}$ electrode before and after 1000 cycles GCD

\begin{tabular}{lcc}
\hline & Fresh electrode & $\begin{array}{l}\text { After } 1000 \\
\text { cycles }\end{array}$ \\
\hline$R_{\mathrm{s}}(\Omega)$ & 1.74 & 1.76 \\
$Q(\mathrm{mF})$ & 2.68 & 2.57 \\
$n$ & 0.86 & 0.87 \\
$R_{\mathrm{ct}}(\Omega)$ & 53.37 & 78.74 \\
$W\left(\Omega \mathrm{s}^{-0.5}\right)$ & 59.67 & 101.90 \\
$C(\mathrm{mF})$ & 35.10 & 84.90
\end{tabular}


layer capacitance. Table 2 reveals that $R_{\mathrm{S}}$, representing the ESR, remains nearly constant even after 1000 GCD cycles. The ESR includes electrolyte resistance, internal resistance of the electrode and contact resistance between the electrode and the current collector. ${ }^{5} R_{\text {ct }}$ suffers a slight increase from $53.37 \Omega$ to $78.74 \Omega$, indicating that the amount of charge stored in this material is slightly decreased. The linear part of the curve is inclined by $\sim 45^{\circ}$, indicating Warburg impedance $(W)$ that is related to the frequency-dependent diffusion resistance of electrolyte ions. ${ }^{15,17,47}$ The value of $W$ increases from $59.67 \Omega$ $\mathrm{s}^{-0.5}$ to $101.90 \Omega \mathrm{s}^{-0.5}$, indicating a slowdown in the diffusion rate due to a somewhat difficult penetration of electrolyte ions through the electrode mesopores with long-term use.

\section{Conclusions}

Mesoporous $\mathrm{MnCoFeO}_{4}$ nanoparticles, with diameters in the range 30-40 nm, were successfully synthesized via a one-pot simple co-precipitation method without annealing as confirmed by the TEM analysis. The XRD results confirmed the spinel structure of the fabricated $\mathrm{MnCoFeO}_{4}$ nanoparticles. The $\mathrm{BJH}$ average pore diameter was found to be $10.036 \mathrm{~nm}$ and the BET surface area was $104.963 \mathrm{~m}^{2} \mathrm{~g}^{-1}$. The as-prepared $\mathrm{MnCoFeO}_{4}$ material was evaluated as a supercapacitor electrode in $6 \mathrm{M} \mathrm{KOH}$ using $\mathrm{CV}$ and GCD measurements. The maximum $C_{\mathrm{sp}}$ calculated from $\mathrm{CV}$ measurement was $675 \mathrm{~F} \mathrm{~g}^{-1}$ at a scan rate of $1 \mathrm{mV} \mathrm{s}^{-1}$ and that calculated from GCD measurement was $670 \mathrm{~F} \mathrm{~g}^{-1}$ at a current density of $1.5 \mathrm{~A} \mathrm{~g}^{-1}$. The energy and power densities of the $\mathrm{MnCoFeO}_{4}$ supercapacitor were $18.85 \mathrm{Wh} \mathrm{kg}^{-1}$ and $337.50 \mathrm{~W} \mathrm{~kg}^{-1}$, respectively at a current density of $1.5 \mathrm{~A} \mathrm{~g}^{-1}$. The cyclic stability was scrutinized via GCD and EIS. The capacitance retention (\%) was $111.43 \%$ and $92.86 \%$ after 200 and 1000 GCD cycles, respectively, indicating an excellent long-term stability. The equivalent series resistance (ESR) remained nearly the same even after 1000 GCD cycles.

\section{Conflicts of interest}

There are no conflicts to declare.

\section{Acknowledgements}

All facilities provided by the American University in Cairo, Egyptian Petroleum Research Institute and Cairo University are greatly appreciated.

\section{Notes and references}

1 E. M. Gabr, S. M. Mohamed, S. A. El-Temtamy and T. S. Gendy, Egypt. J. Pet., 2016, 25, 65-74.

2 L. Hao, X. Li and L. Zhi, Adv. Mater., 2013, 25, 3899-3904.

3 C. Liu, F. Li, L. P. Ma and H. M. Cheng, Adv. Mater., 2010, 22, E28-E62.

4 N. Choudhary, C. Li, J. Moore, N. Nagaiah, L. Zhai, Y. Jung and J. Thomas, Adv. Mater., 2017, 29, 1605336.
5 A. Yu, V. Chabot and J. Zhang, Electrochemical supercapacitors for energy storage and delivery: fundamentals and applications, CRC Press, New York, 2013.

6 F. Béguin, V. Presser, A. Balducci and E. Frackowiak, Adv. Mater., 2014, 26, 2219-2251.

7 D. Chen, Q. Wang, R. Wang and G. Shen, J. Mater. Chem. A, 2015, 3, 10158-10173.

8 D. M. El-Gendy, N. A. A. Ghany, E. F. El Sherbini and N. K. Allam, Sci. Rep., 2017, 7, DOI: 10.1038/srep43104.

9 S. Faraji and F. N. Ani, Renewable Sustainable Energy Rev., 2015, 42, 823-834.

10 K. Bindu, K. Sridharan, K. M. Ajith, H. N. Lim and H. S. Nagaraja, Electrochim. Acta, 2016, 217, 139-149.

11 D. Ham, J. Chang, S. H. Pathan, W. Y. Kim, R. S. Mane, B. N. Pawar, O.-S. Joo, H. Chung, M.-Y. Yoon and S.-H. Han, Curr. Appl. Phys., 2009, 9, S98-S100.

12 B. Bhujun, M. T. T. Tan and A. S. Shanmugam, Results Phys., 2017, 7, 345-353.

13 Y. Zhang, L. Li, H. Su, W. Huang and X. Dong, J. Mater. Chem. A, 2015, 3, 43-59.

14 L. Liu, H. Zhang, Y. Mu, Y. Bai and Y. Wang, J. Power Sources, 2016, 327, 599-609.

15 M. K. Zate, S. M. F. Shaikh, V. V. Jadhav, K. K. Tehare, S. S. Kolekar, R. S. Mane, M. Naushad, B. N. Pawar and K. N. Hui, J. Anal. Appl. Pyrolysis, 2015, 116, 177-182.

16 Y. Zhang, Q.-q. Yao, H.-l. Gao, L.-s. Zhang, L.-z. Wang, A.-q. Zhang, Y.-h. Song and L.-x. Wang, J. Anal. Appl. Pyrolysis, 2015, 111, 233-237.

17 M. M. Vadiyar, S. S. Kolekar, J.-Y. Chang, A. A. Kashale and A. V. Ghule, Electrochim. Acta, 2016, 222, 1604-1615.

18 S. Navale, V. Mali, S. Pawar, R. Mane, M. Naushad, F. Stadler and V. Patil, RSC Adv., 2015, 5, 51961-51965.

19 H. Wu and K. Lian, J. Power Sources, 2014, 271, 534-537.

20 J. Xu, T. Ding, J. Wang, J. Zhang, S. Wang, C. Chen, Y. Fang, Z. Wu, K. Huo and J. Dai, Electrochim. Acta, 2015, 174, 728734.

21 B. Lokhande, R. Ambare and S. Bharadwaj, Measurement, 2014, 47, 427-432.

22 P. Xiong, H. Huang and X. Wang, J. Power Sources, 2014, 245, 937-946.

23 A. Shanmugavani, D. Kalpana and R. K. Selvan, Mater. Res. Bull., 2015, 71, 133-141.

24 Y. Xu, J. Wei, J. Yao, J. Fu and D. Xue, Mater. Lett., 2008, 62, 1403-1405.

25 Y. Asano, K. Nakaoka, K. Murashiro, T. Komatsu and K. Hoshino, Mater. Lett., 2012, 81, 162-164.

26 S. Giri, D. Ghosh, A. P. Kharitonov and C. K. Das, Funct. Mater. Lett., 2012, 05, 1250046.

27 V. V. Jadhav, M. K. Zate, S. Liu, M. Naushad, R. S. Mane, K. N. Hui and S.-H. Han, Appl. Nanosci., 2016, 6, 511-519.

28 M. Li, W. Xu, W. Wang, Y. Liu, B. Cui and X. Guo, J. Power Sources, 2014, 248, 465-473.

29 X.-q. Bao, J. Zhu, X.-x. Gao, Y. Qiao and S.-z. Zhou, J. Magn. Magn. Mater., 2009, 321, 1832-1837.

30 M. Zhu, X. Zhang, Y. Zhou, C. Zhuo, J. Huang and S. Li, RSC Adv., 2015, 5, 39270-39277. 
31 P. R. Graves, C. Johnston and J. J. Campaniello, Mater. Res. Bull., 1988, 23, 1651-1660.

32 J.-L. Bernard, N. Baffier and M. Huber, J. Solid State Chem., 1973, 8, 50-56.

33 D. Kulkarni, Curr. Sci., 1974, 43, 374-375.

34 J. Martens, J. Appl. Phys., 1986, 59, 3820-3823.

35 I. Chassaing, L. Presmanes, P. Tailhades and A. Rousset, Solid State Ionics, 1992, 58, 261-267.

36 M. Laarj, S. Kacim and B. Gillot, J. Solid State Chem., 1996, 125, 67-74.

37 P. Guo, Z. Li, S. Liu, J. Xue, G. Wu, H. Li and X. S. Zhao, J. Mater. Sci., 2017, 52, 5359-5365.

38 Z. Wang, W. Jia, M. Jiang, C. Chen and Y. Li, Nano Res., 2016, 9, 2026-2033.

39 A. Rai, A. L. Sharma and A. K. Thakur, Solid State Ionics, 2014, 262, 230-233.

40 W. Wang, Q. Hao, W. Lei, X. Xia and X. Wang, J. Power Sources, 2014, 269, 250-259.
41 M. A. Mousa, M. Khairy and M. Shehab, J. Solid State Electrochem., 2017, 21, 995-1005.

42 P. Xiong, C. Hu, Y. Fan, W. Zhang, J. Zhu and X. Wang, J. Power Sources, 2014, 266, 384-392.

43 K. S. Sing, Pure Appl. Chem., 1985, 57, 603-619.

44 D. K. Pawar, S. M. Pawar, P. S. Patil and S. S. Kolekar, J. Alloys Compd., 2011, 509, 3587-3591.

45 M. D. Arning and S. D. Minteer, in Handbook of Electrochemistry, Elsevier, 2007.

46 J. Hao, W. Yang, Z. Zhang, B. Lu, B. Zhang and J. Tang, Electrochim. Acta, 2015, 152, 13-18.

47 H. Che, A. Liu, J. Mu, C. Wu and X. Zhang, Ceram. Int., 2016, 42, 2416-2424.

48 S. G. Mohamed, S. Y. Attia and N. K. Allam, Mater. Today Energ., 2017, 4, 97-104.

49 B. Bhujun, M. T. T. Tan and A. S. Shanmugam, Ceram. Int., 2016, 42, 6457-6466. 